\title{
Liaison psychiatry: heart and lung transplantation
}

\author{
John D. Sheehan
}

\begin{abstract}
SUMMARY
Owing to the continuing shortage of organs, the demand for transplantation outstrips supply. Liaison psychiatrists, as part of a multidisciplinary team, have a key role in the assessment of transplant candidates and participate in the decisionmaking process regarding 'listing' the patient for transplantation. Psychiatric and psychosocial factors affect prognosis post-transplantation and there are absolute and relative contraindications to transplantation. The skills of the liaison psychiatrist are employed throughout the patient's illness journey, in the pre-, peri- and post-transplantation periods.
\end{abstract}

\section{LEARNING OBJECTIVES}

- Learn the salient factors in a pre-transplantation psychosocial assessment

- Know the contraindications to transplantation

- Appreciate the factors associated with a poor outcome post-transplantation

\section{DECLARATION OF INTEREST}

None.

Heart transplantation and lung transplantation can be life-saving procedures for patients with endstage heart disease and lung disease respectively. Transplantation may also significantly improve quality of life for patients. However, there is a shortage of organs as the number donated does not match the number required.

The liaison psychiatrist has a key role as a member of the multidisciplinary transplant team. One of the unique features of transplantation psychiatry is that the liaison psychiatrist is involved in the pre-transplant assessment of patients to determine their suitability for transplantation. The liaison psychiatrist also participates in the decisionmaking meeting regarding the 'listing' of a patient for transplant. If the liaison psychiatrist deems the patient to be unsuitable for transplantation, then that decision and the basis for it has to be communicated and explained to the multidisciplinary transplant team. If ethical questions arise in the context of transplantation, the liaison psychiatrist is well placed to contribute to the discussion and decisionmaking process.

The liaison psychiatrist is involved in the diagnosis and management of mental disorders in the pre-, peri- and post-transplantation periods. The presence of psychiatric disorder pre-transplantation is associated with worse physical outcomes with increased morbidity and mortality. Hence, the liaison psychiatrist has to try to help the patient pre-transplantation in order to achieve maximum improvement or recovery after transplantation has taken place.

The time before 'listing' can be very stressful for patients, particularly those who are critically ill and know they will die without a transplant. Similarly, following 'listing', the patient waits for the call that an organ has become available. This can be a very anxious time, especially as the patient knows that there is a chance that they will die before an organ becomes available.

Following transplantation, the expertise of the liaison psychiatrist may be required in the management of a number of disorders, including delirium, adjustment disorders, anxiety disorders and depressive illness, and with drug reactions.

In this article, I consider the pre-, peri- and posttransplantation periods in succession. The subject matter covered relates to heart and lung transplantation, but much of it is applicable to other transplantable organs such as kidney, liver and pancreas. Issues specific to heart and lung transplantation are highlighted.

\section{Pre-transplantation}

\section{The pre-transplantation assessment}

The pre-transplant assessment is done to assess the patient's suitability for transplantation (Boxes 1 $\& 2$ ). The assessment consists of a clinical psychosocial assessment with a full psychiatric history and mental state examination, including cognitive function testing. The patient's attitude to transplantation is explored, along with their current complaints, their own and family psychiatric history, personal history, substance use history and personality. Validated instruments such as the Stanford Integrated Psychosocial Assessment for Transplantation may also be used. The prospective
John D. Sheehan is an associate clinical professor in the School of Medicine at University College Dublin and consultant in liaison psychiatry at the Mater Misericordiae University Hospital, Dublin. The National Heart and Lung Transplantation service is based at the Mater Misericordiae University Hospital.

Correspondence Dr John Sheehan Department of Adult Psychiatry, Mater Misericordiae Hospital, 62/63 Eccles Street, Dublin 7, Ireland. Email: sheehanj@mater.ie

\section{Copyright and usage} (C) The Royal College of Psychiatrists 2018 
BOX 1 Factors considered in the pre-transplantation assessment

- Attitude

- Current complaints

- Psychotropic use

- Own and family psychiatric history

- Adherence

- Personal history

- Substance use

- Social supports

- Mental state examination, including cognitive function and 10

transplant recipient is assessed by the members of the multidisciplinary team. Subsequently, the team members, including the liaison psychiatrist, meet to report their findings and to decide on whether the patient should be put on the transplant list. The liaison psychiatrist is well placed to conduct the psychiatric assessment as they are based in the acute hospital and have regular contact with the relevant medical and surgical teams, along with the allied health professionals. Contact may be made with any general adult or addiction service with which the patient has had prior involvement. Such services provide useful information particularly about substance use and adherence to treatment. The patient's general practitioner may be also contacted to obtain psychosocial information.

\section{Attitude to transplantation}

In the assessment, enquiry is made about the patient's attitude to transplantation. Has the patient received sufficient information about the procedure and its aftermath? What is their knowledge and understanding of transplantation? Have they carefully considered the pros and cons of having or of not having the transplant? Do they understand the requirements for aftercare? Have they thought about the potential donor? Would they proceed if

BOX 2 Relative contraindications to transplantation

- Failure of treatment adherence

- A history of self-harm

- Active psychosis

- Active or recent (within 6 months) substance misuse

- Significant cognitive impairment

- Significant intellectual disability $(10<70)$ offered a transplant? This exploration also covers aspects of informed consent. Some patients are suitable surgically for transplantation, but decline the transplant for personal reasons. A proportion of patients become ambivalent after receiving transplant-related information. It is important to allow sufficient time for the patient to process the difficult and complex material. The ambivalence may not be related to educational level. With good support, patients with virtually no literacy can engage well and understand the complexities of transplantrelated material. However, in my experience, when a patient is very sick physically with a reduced life expectancy and poor quality of life, they usually request a transplant. Patients know they have few options.

\section{Current complaints}

The liaison psychiatrist elicits current psychiatric symptoms, if any, along with any psychotropic use. The patient may have anxiety or depressive symptoms. In adult patients with cystic fibrosis, the prevalence of anxiety is higher whereas the prevalence of depression is lower than in the general population (Goldbeck 2010). However, patients with end-stage lung disease other than cystic fibrosis, namely emphysema and idiopathic pulmonary fibrosis, have higher pre-transplantation anxiety scores than those with cystic fibrosis, possibly because cystic fibrosis patients have better social supports and coping strategies (Burker 2000). Any identified mental illness is treated - a comprehensive management plan is put in place. However, the presence of active psychotic symptoms is regarded as a relative contraindication to transplantation.

\section{The patient's and family's psychiatric history}

The patient's and the family's psychiatric history can be crucial in the decision to 'list' a patient for transplantation. Family history is useful in terms of raising awareness of potential risk factors.

Enquiry is made about psychiatric history, including details of diagnoses made, the severity and chronicity of illness, functional impact, treatment received and response to treatment. In particular, details of contact with mental health services, the general practitioner, previous admissions, out-patient attendances, medication prescribed and therapies undertaken are all documented. Adherence to treatment is carefully evaluated in terms of attendance at clinic appointments, medication use and engagement with psychologists or therapists. An opinion has to be formed as to the patient's ability and willingness to collaborate with the transplant team and comply with the medical 
regime. Furthermore, an opinion is made about the patient's ability to cope with the surgery, a major life event. Failure of treatment adherence is regarded as a relative contraindication to transplantation.

A recent suicide attempt or a history of repeated suicide attempts are generally regarded as absolute contraindications to transplantation (Levinson 2000), whereas a remote history of a suicide attempt is a relative contraindication.

A history of self-harm is also deemed to be a relative contraindication to transplantation. Has the patient a personality disorder? Has the patient a history of impulsivity and unstable moods? Was the self-harm a suicide attempt? Was it impulsive or planned? What was the reason for the selfharm? What is the likelihood of another attempt, particularly if the patient receives a transplant? These questions need to be addressed. Clinical judgement is required. Generally, the transplant team will be reluctant to perform a transplant if the patient has a history of self-harm. The liaison psychiatrist may need to take on a patient advocate role if appropriate.

\section{Personal history}

In personal history, an assessment of functioning, ability to live independently and the level of supports available to the patient is crucial. Careful evaluation is required. Furthermore, evaluation of personality is relevant because of risk-taking, adherence issues and stability of support systems. Enquiry is made about religious beliefs and practice, as well as involvement in community-based activities.

\section{Substance use}

The substance use (alcohol and drug) history needs careful exploration. Substance use itself may be a cause of organ failure, as in cocaine-related dilated cardiomyopathy, or it may be incidental. Substance misuse is associated with poor adherence pre-transplantation and is a risk factor for nonadherence post-transplantation (Surman 1992). Substance dependence can lead to complications post-transplantation such as a withdrawal state that could jeopardise the success of the transplant.

Active alcohol misuse, ongoing dependence or any illicit drug use are generally regarded as contraindications to transplantation. The consensus is that a patient with alcohol or drug misuse or dependence must be abstinent from alcohol or drugs for 6 months before being listed for transplantation. Generally, the history given by the patient is sufficient in evaluating substance use. However, if there is any uncertainty or if the history does not seem to match clinical findings, then a collateral history is required. If a patient is dependent on a substance, then I insist on the patient being abstinent, attending an addiction service and providing supportive evidence from the addiction team/counsellor of abstinence prior to being listed for transplantation. The supportive evidence may include random blood or urine testing or even hair sampling.

There is debate about the clinical significance of cannabis use by candidates. Is a patient who uses cannabis in small quantities, perhaps only at weekends, suitable or unsuitable for transplantation? Clinical judgement is required on a case-by-case basis and discussion at the multidisciplinary transplant team meeting is required.

Substance misuse may be hidden. A detailed alcohol history is required for patients with 'idiopathic' cardiomyopathy. A high index of suspicion is required for prescribed drug misuse or dependence. Hidden opiate dependence may be present in a patient with ischaemic cardiomyopathy prescribed regular opiates for recurring chest pain. Similarly, benzodiazepine dependence may be present in an anxious patient with chronic lung disease. Undetected dependence can lead to a withdrawal state post-transplantation and complicate post-operative care and recovery.

\section{Mental state examination}

The initial assessment provides a baseline of mental state which is useful when following the patient through their illness journey. Later mental state examinations can be compared with the initial examination. Certain findings set off alarm bells. Active psychotic illness is a relative contraindication to transplantation. However, clearly there is a major difference between an acute psychosis that is likely to respond to treatment and a chronic and severe illness, with functional impairment, that may be treatment resistant.

There is no universal agreement as to whether the presence of mental illness is an absolute or relative contraindication to transplantation. Patients with illnesses such as bipolar disorder that is well controlled on lithium and who are adherent to their treatment can do very well post-transplantation. Consequently, it is the severity, chronicity and response to treatment of an illness that have to be considered.

If cognitive deficits are suspected that could impair self-care and adherence post-transplantation, specific neuropsychological testing may be required. The expertise of a neuropsychologist is needed and neuropsychological intervention may be helpful. Certain patient groups are at higher risk of specific deficits: a patient with vascular disease and ischaemic cardiomyopathy may have cognitive deficits such as those associated with a 
vascular dementia. Significant cognitive impairment is a relative contraindication to transplantation.

An estimation of IQ may be required if intellectual disability is present. An IQ of less than 70 is considered a relative contraindication to transplantation. However, what matters is the patient's ability to function and live independently, as well as the level of supports available to them.

\section{Validated instruments}

There are several instruments to measure pretransplant psychosocial factors and likely treatment adherence (Box 3). The Stanford Integrated Psychosocial Assessment for Transplantation (SIPAT) (Maldonado 2012) is a comprehensive screening tool designed to standardise the psychosocial assessment of organ transplant candidates. It encompasses 18 psychosocial risk factors covering the following four domains: the patient's readiness level and illness management; social support system; psychological stability and psychopathology; and lifestyle and effect of substance use. It is scored from 0 to 115, with higher scores indicating higher risk. It has been used in heart, lung, liver and kidney transplant candidates. Other, older tools used include the Psychosocial Assessment of Candidates for Transplantation (PACT) (Olbrisch 1989) and the Transplant Evaluation Rating Scale (TERS) (Twillman 1993). The PACT has eight subscales as well as an initial and final rating. It has four domains: social support; psychological health; lifestyle factors; and understanding of transplant and follow-up. The TERS covers seven domains: substance misuse; adherence; health consciousness; social support; past coping; dealing with the current disease; affect; and mental status. I think that the clinical evaluation is enhanced by the use of an assessment tool.

With regard to personality assessment, validated instruments such as the Minnesota Multiphasic Personality Inventory (MMPI) may be used if personality disorder is suspected, particularly if adherence is an issue.

BOX 3 Validated instruments used pretransplantation

- Stanford Integrated Psychosocial Assessment for Transplantation (SIPAT)

- Psychosocial Assessment of Candidates for Transplantation (PACT)

- Transplant Evaluation Rating Scale (TERS)

- Minnesota Multiphasic Personality Inventory

\section{Assessment and prediction of outcome}

Careful pre-transplantation assessment is important because psychosocial factors may have a significant bearing on the medical outcome post-transplantation. The prognosis for those with psychiatric disorder is worse post-transplantation. In a study by Owen et al (2006), 108 heart transplant recipients were followed for an average of 970 days. It was hypothesised that psychiatric risk factors (e.g. recent substance misuse, history of suicide attempt, having a personality disorder, low levels of social support, poor past adherence to medical regimens) would be associated with a greater likelihood of post-transplantation complications, including re-admission to hospital, organ rejection, infection and death. The study found that increasing psychiatric risk classification was associated with a greater hazard of post-transplant mortality, but not of infection or re-admission. However, a history of suicide attempt was strongly associated with time to infection or rejection.

The detection of mental disorders pre-transplantation is also relevant to psychosocial outcome. Chacko et al (1996) found that Axis I disorders were associated with poorer psychosocial adjustment and health status, whereas Axis II diagnoses were associated with poorer adherence post-transplantation.

Scores obtained using the SIPAT have been used to predict medical and psychosocial outcomes posttransplantation. At 1-year follow up, the higher the scores on the SIPAT the greater the adverse medical and psychosocial outcomes (Maldonado 2015). Higher scores predicted higher rates of rejection episodes, medical admissions, infection rates, psychiatric decompensation and support system failure.

\section{Peri-transplantation}

\section{Bridge to transplant}

One of the unique features of heart and lung transplantation is 'bridging'. Bridging refers to a wide range of intensive care measures that try to stabilise a patient who is in an imminently life-threatening situation. One example is an extracorporeal membrane oxygenation (ECMO) system, which enables direct oxygenation of the blood or $\mathrm{CO}_{2}$ extraction from the blood. Bridging is regarded as a shortterm measure while an organ is awaited. It can be very stressful for the patient, family and staff. A patient can be 'listed' for transplantation but be too sick to be discharged, be on maximum medical therapy and be deteriorating physically while awaiting a transplant. Such patients may openly express their fears of dying to nursing staff, who may feel helpless and guilty about not being able to do 
more to save their life. Such situations are challenging. Psychological support for the patient is essential. For hospital-bound patients, online support groups, apps and other online therapies may be useful. Similarly, individual psychotherapy, antidepressants or anxiolytics/hypnotics may be required. However, with a critically ill patient, decisions about benzodiazepine use are complex, such as when to prescribe anxiolytics without causing respiratory depression in an anxious breathless patient with severe lung disease. The clinical expertise of the liaison psychiatrist is useful in determining when and what to prescribe, and the intervention can allay the fears of the treating medical team.

\section{Post-transplantation}

Mental health problems are common after transplantation. It is widely recognised that such problems impair quality of life and increase physical morbidity and mortality. In a study of cardiac transplant patients, Dew et al (1996) found that, in the first post-transplant year, the prevalence of major depression was $17.3 \%$, post-traumatic stress disorder related to the transplant occurred in 13.7\% and adjustment disorders were found in 10\%. Risk factors identified included: pre-transplant psychiatric history; poor social support from primary family caregiver, other relatives and friends; the use of avoidance coping strategies for managing health problems; and low self-esteem early posttransplant.

In a later study, Dew et al (2000) examined heart transplant recipients over a longer time period - the first 3 years post-transplantation. They found that the cumulative risks for disorder onset were: $25.5 \%$ for major depressive disorder; $20.8 \%$ for adjustment disorders (17.7\% with anxious mood); and $17 \%$ for post-traumatic stress disorder related to the transplant. Of interest, they found that later episodes of depression were more likely to be precipitated by non-transplant-related stressors. Factors increasing the cumulative risk of psychiatric disorders post-transplant included pre-transplant psychiatric history, female gender, longer hospitalisation, more impaired physical function and lower social supports. The risk factor effects were additive - the presence of an increasing number of risk factors bore a dose-response relationship to cumulative risk of disorder.

Dew et al (2012) also studied lung transplant patients. In the first 2 years following transplantation, panic disorder occurred in 18\%, which was significantly higher than in heart transplant patients $(P<0.05)$. They found major depression in 30\%, post-traumatic stress disorder in $15 \%$ and generalised anxiety disorder in $4 \%$. Risk factors were similar to those for heart transplant patients, namely pre-transplant psychiatric history, female gender, longer wait for transplant, early post-transplant health problems, poor caregiver support and use of avoidant coping.

Box 4 lists the factors most commonly associated with poor outcomes following organ transplantations in general.

Most patients are very appreciative of the donor and his or her family for donating the organ. They know that someone had to die so that they could live. However, some patients use denial to cope and avoid thinking about the donor and the graft. The denial is adaptive in nature. Bunzel et al (1992) found that, among recipients of a heart transplant, $82 \%$ accepted the donor heart immediately as their own whereas the remaining $18 \%$ coped using denial.

Questions have been raised about the effect on the recipient's body image of having another person's heart or lungs inside them. Hartmann et al (2017) found that the majority of heart transplant recipients did not experience body image disturbance. In practice, owing to the gratitude experienced by the recipient, body image disturbance is not common.

\section{Drug treatment}

There is a paucity of published data on the safety of psychotropic medication in transplant recipients. If medication is required, the choice of drug needs to be considered carefully. What is safe to use? What dose is appropriate? What side-effects are likely? What drug interactions might occur? How significant are the altered pharmacokinetics? Selective serotonin reuptake inhibitors (SSRIs) are suitable for major depression and panic disorder in heart and lung transplant patients. However, as many SSRIs are metabolised by the same cytochrome P450 isoenzyme system as cyclosporine, elevated cyclosporine levels can occur. Certain psychotropics, especially nefazodone and fluvoxamine, should be avoided because of their interactions with calcineurin inhibitors. Cyclosporine levels should be monitored. However, given that the patient will be on a multiplicity of medications, I think that the

\section{BOX 4 Factors associated with poor post-} transplant outcomes

- Poor social support

- Ongoing psychiatric disorder

- Ongoing substance misuse

- Personality disorder

- Poor treatment adherence 
MCO answers

1 e 2 d 3 e 4 b 5 c best policy is to confer with the hospital pharmacist on potential drug interactions.

Immunosuppressants such as cyclosporine and tacrolimus are associated with neuropsychiatric side-effects. Cyclosporine is associated with delirium and anxiety and may elevate serum lithium levels.

Corticosteroids, particularly at high doses, may be associated with a wide range of behavioural and psychiatric symptoms, from mild elation to frank psychosis. Steroids may destabilise pre-existing mental health problems. Advice may be sought regarding the use of steroid-sparing or steroidavoiding regimes. The management has to be decided on a case-by-case basis. Drug treatment is determined by the patient's need for steroids, the availability of other treatment options, and the patient's clinical presentation and history. All need careful consideration.

Liaison psychiatrists may be asked to assist in the management of post-operative delirium because of their expertise in the use of antipsychotics. Low doses of typical antipsychotics such as haloperidol or atypicals such as risperidone or quetiapine can be used. If a benzodiazepine is required, a shortacting one with no active metabolites, such as lorazepam, is appropriate.

\section{Psychology}

Psychologists should be part of the multidisciplinary transplant team. Working with liaison psychiatrists, a psychologist may be involved in pre-transplant assessment, particularly if a neuropsychological assessment is required where a patient has a cognitive deficit. Depending on their training, psychologists may do mindfulness or use relaxation techniques with patients on bridging. Psychologists frequently have particular skills such as in the delivery of cognitive-behavioural therapy for depression. They may assist where adherence is an issue. Collaborative working between psychology and liaison psychiatry delivers a better service for the patient.

\section{Family and carers}

The carers and family of transplant candidates and recipients experience significant anxiety and worry. Psychological support is usually provided by the transplant team and specifically by a medical social worker or psychologist. In particular, the emotional strain is considerable among relatives of patients with cystic fibrosis (Burker 2000).

\section{Other areas}

This article has not addressed the issues pertaining to paediatric transplantation, which requires the input of a paediatric liaison psychiatrist.
As part of a multidisciplinary team, the liaison psychiatrist has the opportunity to educate and train other team members regarding the psychological care of transplant patients. Collaborative working with nurses, psychologists, pharmacists, physiotherapists, social workers and other members of the multidisciplinary team ensures the best outcome for the patient.

Research is required in many areas because of our gaps in knowledge and information. Transplantation is evolving: newer surgical treatments are available; criteria for 'listing' need to be refined; prognostic factors need further evaluation. Multicentre outcome studies could provide much needed information.

\section{Conclusions}

The demand for donor organs continues to outstrip supply, and each potential recipient undergoes a pre-transplantation multidisciplinary assessment of suitability for 'listing'. As psychiatric and psychosocial factors affect prognosis, liaison psychiatrists assist in these assessments and participate in the selection process. They also provide psychiatric support for patients in the peri- and post-transplantation periods, and can play an important role in staff education, training and emotional support.

\section{References}

Bunzel B, Wollenek G, Grundböck A (1992) Living with a donor heart: feelings and attitudes of patients toward the donor and the donor organ. Journal of Heart and Lung Transplantation, 11: 1151-5.

Burker EJ, Carels LF, Thompson L, et al (2000) Quality of life in patients awaiting lung transplants: cystic fibrosis versus other end-stage lung diseases. Pediatric Pulmonology, 30: 453-60.

Chacko RC, Harper RG, Kunit M, et al (1996) Relationship of psychiatric morbidity and psychosocial factors in organ transplant candidates. Psychosomatics, 37: 100-7

Dew M, Roth L, Schulberg H, et al (1996) Prevalence and predictors of depression and anxiety-related disorders during the year after heart transplantation. General Hospital Psychiatry, 18: 48S-61S.

Dew M, Kormos R, DiMartini A, et al (2000) Prevalence and risk of depression and anxiety-related disorders during the first three years after heart transplantation. Psychosomatics, 42: 300-13.

Dew M, DiMartini A, DeVito Dabbs A, et al (2012) Onset and risk factors for anxiety and depression during the first two years after lung transplantation. General Hospital Psychiatry, 34: 127-38.

Goldbeck L, Besier T, Hinz A, et al (2010) Prevalence of symptoms of anxiety and depression in German patients with cystic fibrosis. Chest, 138 : 929-36.

Hartmann A, Heilmann C, Kaps J, et al (2017) Body image after heart transplantation compared to mechanical aortic valve insertion. International Journal of Psychiatry in Clinical Practice, 15: 1-6.

Levinson JL, Olbrisch ME (2000) Psychosocial screening and selection of candidates for organ transplantation. In The Transplant Patient: Biological, Psychiatric, and Ethical Issues in Organ Transplantation (eds PT Trepacz, AF DiMartini): 21-41. Cambridge University Press.

Maldonado JR, Dubois HC, David EE, et al (2012) The Stanford Integrated Psychosocial Assessment for Transplantation (SIPAT): a new tool for the psychosocial evaluation of pre-transplant candidates. Psychosomatics, 53: $123-32$ 
Maldonado JR, Sher Y, Lolak S, et al (2015) The Stanford Integrated Psychosocial Assessment for Transplantation: a prospective study of medical and psychosocial outcomes. Psychosomatic Medicine, 77: 1018-30.

Olbrisch M, Levenson J, Hamer R (1989) The PACT: a rating scale for the study of clinical decision making in psychosocial screening of organ transplant candidates. Clinical Transplantation, 3: 164-9.

Owen J, Bonds C, Wellisch D (2006) Psychiatric evaluations of heart transplant candidates: predicting post-transplant hospitalizations, rejection episodes, and survival. Psychosomatics, 47: 213-22.
Surman 0, Portillo R (1992) Reevaluation of organ transplantation criteria. Psychosomatics, 33: 202-12.

Twillman R, Manetto C, Wellisch D, et al (1993) The Transplant Evaluation Rating Scale. A revision of the psychosocial levels system for evaluating organ transplant candidates. Psychosomatics, 34: 144-53.

\section{MCOs}

Select the single best option for each question stem

\section{Pre-transplantation assessment includes} the following:

a adherence to medication

b substance use

c psychotropic use

d psychiatric history

e all of the above.

2 Contraindications to transplantation include:

a 10 over 90

b mild cognitive deficits

c reduced social support

d recent and repeated suicide attempts

e remote history of a single episode of self-cutting.

\section{Validated instruments used pre-transplant-} ation include:

a Stanford Integrated Psychosocial Assessment for Transplantation

b Minnesota Multiphasic Personality Inventory

c Psychosocial Assessment of Candidates for Transplantation

d Transplant Evaluation Rating Scale

e all of the above.

4 Poor outcome post-transplantation is associated with:

a good family support

b poor adherence

c remote history of substance misuse

$\mathrm{d}$ bed-wetting as a child

e male gender.
5 The following is more common in lung than in heart transplant patients:

a adjustment disorder

b depressive episode

c panic disorder

d generalised anxiety disorder

e personality disorder 